\title{
NOISE REDUCTION IN CDMA SYSTEM USING SOFT HANDOVER
}

\author{
Raad H. Thaher \\ Ministry of Higher Education and Research-Baghdad-Iraq \\ Email:raadthaher@yahoo.com
}

\begin{abstract}
Mobile cellular communication becomes more and more important in today's communication. In cellular network, the radio and fixed links required are not permanently allocated for the duration of a call. The function of the handover is to make an on going call to different cell or channel. Also it maintains a call in progress across cell boundaries in mobile communication. ESTI (European Telecommunications standards Institute) adopted Wideband code division multiple access as the mainstream air interface solution for the third-generation networks to provide a multitude of services, especially multimedia and high -bit-rate packet data.
\end{abstract}

In this paper the soft/softer handover and hard handover for voice and packet data in WCDMA systems are presented. Hard handover is necessary in WCDMA and will be presented in this paper. Softer handover is implemented within one base station between different sectors; it doesn't cause the system load increase as implemented with in the base station. The implementation of soft handover can increase the coverage, capacity performance or balance the network load. Important parameters such as, adding threshold, dropping threshold and timer-to-dropping will be analyzed.

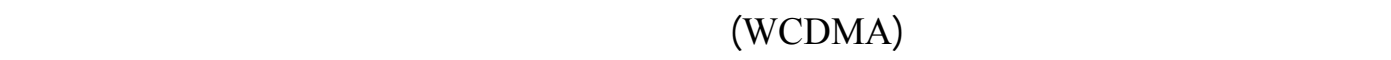

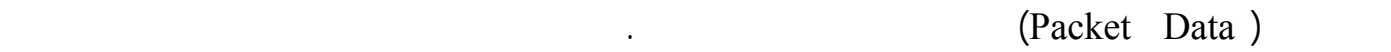

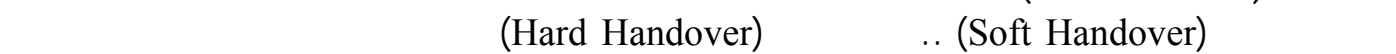

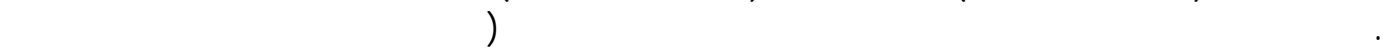

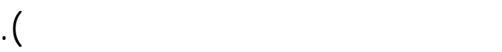

Keywords: Softer Handover, Hard Handover.

\section{INTRODUCTION}

Third generation mobile radio networks, $3 \mathrm{G}$, have been under intense research and discussion and emerge around the year2000.In the international Telecommunications Union (IUT), third generation networks are called International Mobile Telecommunication System-2000(IMT-2000), and in Europe, Universal Mobile Telecommunications System(UTMS) .IMT-2000 provide a multitude of services, especially multimedia and high- bit- rate packet data.WCDMA has emerged as the mainstream air interface solution for the third generation networks.

WCDMA allow very high- speed multimedia services such as voice, Internet access and videoconferencing. In order to provide the

multiple services, handover is essential for seamless communication. Handover is one of the mechanisms that transfer an ongoing call from one cell to another as a user moves through the coverage area of a cellular. There are two types of handover: hard handover and soft handover. Hard handover is a break -before-make method, where a new channel is set up after the release of the old channel. A certain amount of margin may be introduced to eliminate the ping-pong effect. Hard handover is supported by TDMA, FDMA, and CDMA systems.

In WCDMA all users in the same cell share the same frequency spectrum simultaneously. In a WCDMA based cellular network, this is true for users in different cells. This makes that WCDMA also support soft handover, which is a make-before-break method. When the pilot signal from a new base station (BS) is stronger than the threshold value, a new link to the BS is established while maintaining the existing link. In this case the call is said to be in soft handover. Handover is needed in two situations where the cell site receives weak signal from the mobile unit (1) at the cell boundary, $-100 \mathrm{~dB}$ for example, which is the level for requesting a handover in a noise -limited environment; and(2) when the mobile unit is reaching the signal -strength holes(gaps) within the cell site. The number of handover, adding threshold and dropping threshold of pilot signal, timer are the important parameters in handover for system performance. The number of handover depends on the adding threshold and 
dropping threshold of pilot signal and timer. The increasing number of handover causes ping-pong phenomenon. As a smaller cells are deployed to meet the demands for increased capacity, the number of cell boundary increases. Each handover requires network resources to reroute the call to the new base station .The switching load can be minimized by minimizing the number of handovers. If handover does not occur quickly, the quality of service (QoS) may degenerate below an acceptable level and there is a brief service interruption. As the frequency of these interruptions increases the perceived QoS is reduced and the chances of dropping a call due to factors such as the availability of channels increase with the number of handover attempts. All these issues promote finding out a set of optimal parameter values of handover in order to provide the QoS in WCDMA. Fortunately, the signal structure of WCDMA is well suited for implementation of soft handover. The universal frequency reuse enables the new function soft handover. The connection between a MS(Mobile station) and the core network can include several radio links. In the up link, two or more base stations can receive the same signal because of the reuse factor of one. In the down link the mobile station can coherently combine the signals from different base stations since it sees them as just additional multipath components. This provides an additional benefit called macro diversity (i.e, the diversity gain provided by the reception of one or more additional signals).A separate channel called pilot is usually used for the signal strength measurements for handover purposes.

\section{HANDOVER CLASSIFICATION IN WCDMA SYSTEM}

Handover in WCDMA can be classified as hard handover and soft handover ${ }_{[1],[2] .}$ In handover based systems such as a GSM, the mobile station keeps the connection to only one base station at a time. breaking the connection to the former base stations immediately before making the new connection to the target base station.In soft handover scheme, the new link between the mobile station and the target base station is built before breaking the old link from the base station.

Depending on the different applications, soft handover can be classified into handover for voice or for packet data transmission. In soft handover a mobile station is connected to more than one base station simultaneously for a period of time. Figure (1) shows the soft handover situation. Soft handover is used in WCDMA to reduce the interference into other cells in power. It improves performance through macro diversity. Softer handover is a soft handover between two sectors of a cell. Soft handover causes strict requirement to power control and makes capacity enhancement by adding a new cell very easy as no frequency planning is necessary. We emphasize on soft handover for voice and soft handover for non real time data and introduce the softer handover within WCDMA system.

There are two types of Handover in WCDMA system the intra-frequency handover and inter-frequency handover by channel frequency mobile station uses. if the channel uses the same frequency after handover finishes as that one used before handover proceed, this is called intra-frequency handover, otherwise, if the channel uses the different frequency after handover finishes with that one used before handover proceed, this is called interfrequency handover ${ }_{[3]}$.

Intra-frequency handover also includes intra-mode, inter-mode and inter-system handover ${ }_{[3]}$.It is a hard handover by which the link between mobile station and base station is temperorarily disconnected. Interfrequency handovers can be used, for example, to hand a mobile over from one WCDMA frequency carrier to another to maximize the use of several carriers per base station.

The intra-mode handover of inter -frequency in WCDMA system is the handover between different channels within one cell or one sector or among macro-cell, micro-cell or Pico-cell in a hierarchical network structure in order to improve coverage and capacity or to balance the loading network. The intermode handover of inter-frequency is the handover between WCDMA system and multi-carrier CDMA. The inter-system handover of inter-frequency is the handover between WCDMA FDD system and another system, such as WCDMA TDD or GSM system.

\section{SOFTER HANDOVER}

Softer handover means that mobile station changes its channel from one sector to another sector within one cell .It provides additional diversity gain and doesn't cause extra transmission between base station and mobile station ${ }_{[3]}$. Figure (2) shows a basic softer handover .In the down link communication, when a mobile is in the overlapping area of two adjacent sectors for one cell of a base station. There are two physical connections between base station and mobile station via two interface channels, one for each sector separately because WCDMA uses orthogonal variable spreading factor codes to distinguish different downlink physical channels, this leads to a softer handover to use two separate codes in the downlink direction, so that the mobile station can separate the signals, it's similar to multipath reception, the only difference is that the f needs to generate the respective code for each sector for the appropriate operation. Holma ${ }_{[3]}$ gave a detailed information. In the uplink direction, base station uses the same method as Mobile station does in the downlink to combine the two received signals from 
two sectors .The two received signals are Maximal Ratio Combined (MRC) in base station .When a user is in overlapping region as shown in figure(2), if the addition of signal levels received by two adjacent sectors is higher than signal level received by the nearest other-cell sector, the user is in softer handover and is power controlled by both sensors $[4]$. The softer handover can improve system performance and during a softer handover, only one power control is active ${ }_{[3]}$. The disadvantage of softer handover is that it creates additional interference when the increase in the effective receiving angle enlarges overlapping region and causes more interference to the user.

\section{SOFT HANDOVER PRINCIPLE IN WCDM}

In soft handover, a mobile station is allowed to be simultaneously connected to several base stations, which are selected by using relative threshold. Figure (4) shows the soft handover principle with two base stations involved. A mobile station enters the soft handover state when the signal strength of neighboring cell exceeds a certain threshold but is still below the current base station's signal strength. Soft handover in WCDMA is a kind of intrafrequency handover. Soft handover is one of the essential features because of its suitability for CDMA based mobile systems.Therefore, soft handover became one of the main issues and key challenges for WCDMA system . Soft handover promises better performance than hard handover. The two most wellknown benefits are fade margin improvement and higher uplink capacity, while the disadvantages include higher down link interference and complex implementation. Proper design of handover is one of the main challenges in mobile communications, since it has a great impact on the system performance and capacity $_{[5]}$. In the uplink direction, each base station in the active set receives simultaneously the signal from the same mobile station because of the frequency reuse. The base station decodes the signal and after decoding a frame, the base station sends the frame to Radio Network Controller (RNC), which choose the base station with the best signal quality on a frame basis and sends the data further. Hence it is different from softer handover in this respect. In Fig(3) two base stations receive the uplink signal from the mobile station simultaneously and demodulate the signal respectively and pass the signal to the combining point,e.g the base station controller.

In the downlink direction, mobile station receives signals from certain number of base stations simultaneously and coherently combine them into one signal by maximal ratio combining method since it sees them as just additional multipath components and this provides an additional benefit called macro diversity ( the diversity gain provided by the reception of one or more additional signals). In Fig.3 the two base stations in the downlink direction transmit the same information, and the mobile receives the signals from the two base stations simultaneously as a separate multipath signals and combine them by maximal ratio combined(MRC).

With soft handover technique, when the algorithms parameters are designed properly, each MS can potentially use the multiple radio links simultaneously, it may receive its signals from the multiple BSs and, generally, the same BSs then receive the signal which is transmitted from the MS. The near far effect is avoided. WCDMA system capacity is substantially improved and the coverage is extended .The coverage size of a cell is adjusted dynamically according to the interference from other cells. The system load is balanced among cells the macro diversity offered by soft handover is against shadowing fading and fast fading. Soft handover is an essential interference mitigating tool in WCDMA.The gain of soft handover in the downlink depends on the gain of macro diversity and the loss of performance due to increased interference. In the uplink, it occupies more physical channels, it needs more radio resources to be allocated to give extra transmission across the interface between the base station and radio network comtroller, this is different than softer handover.

\subsection{Soft Handover Algorithm}

A soft handover algorithm works with the cooperation of a set of system parameters such as adding threshold, dropping threshold and dropping timer. In principle, if the received signal strength from a new base station is higher than adding threshold, it is added into the user's active set and starts the communication to the user. The loss of connection to the user occur when the signal strengthfrom the base station is lower than the dropping threshold for the period of dropping threshold ,therefore,it is removed from the active $\operatorname{set}_{[3]}$. Hiltumen [6] showed a possible handover algorithm. The primary common pilot channel (CPICH) in WCDMA is used to measure the signal strength for soft handover. Each cell or sector has only one primary common pilot channel under the primary scrambling code with a fixed channel code allocation. The CPICH Ec/No is the received energy per chip divided by the power density in the band, the reference point is the antenna connector at the mobile station. Fig (4) shows the general schematic diagram of the WCDMA sift handover algorithm and shows the basic idea of soft handover algorithm. The $\mathrm{CPICH} \mathrm{Ec} / \mathrm{No}$ used by soft handover algorithm is signaled to radio network controller (RNC) by using layer 3 signaling. The algorithm can be described by the Radio link addition (defined as Event 1A ), Radio link removal (defined as Event 1B ), and the 
combined radio link addition and removal (defined as Event 1C). Where

Reporting_range $=$ the threshold for soft handover Hysteresis_event $1 \mathrm{~A}=$ the addition hysteresis Hysteresis event $1 \mathrm{~B}=$ the removal hysteresis Hysteresis event $1 \mathrm{C}=$ the replacement hysteresis $\Delta \mathrm{T}=$ the time to trigger

Different designs of these parameters can form different handover algorithms that have different effects on the system performance. Yang etal ${ }_{[5]}$ classifies soft handover algorithms into 4 types according to the fixed adding threshold and dropping threshold or dynamic adding threshold and dropping threshold used in the algorithms. In WCDMA system, soft handover decision algorithm is located at RNC . Relative threshold causes less interference sensitivity situation than absolute threshold. The setting of these thresholds significantly affects the trade off between coverage, capacity and quality in system. The relative threshold value should be adaptively set by traffic load of the system in order to reduce the interference.Binucci [7] analyzed the influence of the threshold value to the system through simulating the system.

The accuracy of measured $\mathrm{CPICH} \mathrm{Ec} / \mathrm{No}$ is important for soft handover because an accurately measured value will cause the ping-pong effect or degrades the quality of the received signal. The filtering length should be long enough to avoid the fast fading.

The soft handover may highly alter the quality of service; WCDMA system capacity and performance depending on the system load. On the other hand, if mobile make handover with high rate, the network will suffer a high signaling load, this nullifies the soft handover gain if the handover rate is very high. Time-to-trigger mechanism is used to modify measurement reporting behavior compromise between the high signaling load and degradation. To limit the measurement signaling load, a time to trigger parameter could be connected with each reporting event (addition time for event 1A, Deletion time for event $1 \mathrm{~B}$ and replacement time for event 1C).

We should compromise the number of soft handover and the system degradation. We should also consider the different loading situations of the system and the trade off between system capacity and the signaling load when we set the handover timers. The time setting influence to the signaling load is analyzed through the simulating the network under a certain assumptions ${ }_{[8]}$. As a case study in the down link, this paper shows a big difference of the system performance with the different density of active user changes.
Interference in WCDMA system depends on the number of the active users, i.e the load of the system. So when the load is increases, the system interference increases, it is important for the proper timer setting to estimate the interference correctly. Simulation ${ }^{(8)}$ shows that when the timer equal or less than $4 \mathrm{~s}$ with 20 users the degradation of the system is tolerable, which means that we can set the optimal timer in the cellular system to decrease the signaling load. In a higher load case,e.g 40 users, two-second as the timer is suitable. Fig 5 shows the basic mechanism for time to trigger. In order to modify measurement reporting behavior, it is necessary to adjust a cell border by the set individual offsets, it is an efficient way for the network to change the reporting of an individual primary CPIPH. Fig. 6 shows the offset for CPICH 3 in order to extend its border. Total CDMA traffic is the sum of traffic due to conversations (conversation traffic), and additional traffic due to soft handover overhead. In order to reduce the system loading, it is necessary to avoid the un- necessary handover caused by the unstable cells. In Fig.7, PCHICH3 is forbidden to affect the reporting ranges as its quantity is quite unstable. Soft handover gain can be achieved through firstly RNC selects the best frame from base station in the active set on a frame by frame basis ,secondly fast power control doesn't compensate for deep fading. Siplia ${ }_{[9]}$ analyzed the soft handover gains in a fast power controlled WCDMA uplink.

\section{THE TIMING FOR SOFT HANDOVER}

The timing of active set is also important for soft handover.Fig.8shows the timing measurement for soft handover. In the downlink, soft handover gain is acquired with MRC by mobile stations. However, WCDMA is an asynchronous system network, so it is necessary to adjust the transmission timing among the active sets. It allows coherent combining in the RAKE receiver to get multipath diversity, otherwise, it will be difficult for the mobile station to combine the receiving signals from the different base stations.Holma $_{[3]}$ analyzed the timing adjustment in detail. The new base station adjusts the downlink timing in steps of 256 chips based on the information it received from the RNC.

\section{HARD HANDOVER}

\subsection{Intrafrequency handover}

Shared channels and common channels, can't use soft handover; they support hard handover when needed. Decision procedure of this type of handover is the same as soft handover controlled by Network Evaluated Handover (NEHO) and RNC, and causes temporary disconnection of the user.

\subsection{Interfrequency Hard Handover}

WCDMA has 20MHZ bandwidth, it can be split into smaller multiple bandwidth with different carrier 
transmission, so each cell in WCDMA network will have multiple frequency carriers. For a hot spot cell, it could have more numbers of frequencies than the neighboring cells. Furthermore, in order to give a good coverage, the hierarchical cell structure will be used. For this kind of structure, micro cells will have different frequency channels than the macro cells overlying the micro cells. It is also important for WCDMA to handover between different frequencies, it can be a hard handover within one base station or within one RNC, or between different RNC.So an efficient procedure is needed for this type of handover. For GSM or IS-95 system, an efficient method for iter-frequency handover are needed for measuring signal strength and quality on other frequencies while still having the connection running on the current frequeny.Prasad ${ }_{[10]}$ gave a view for inter-frequency handover, where two methods were analyzed for inter-frequency measurement in WCDMA . They are

\section{a-Dual receiver \\ b- Slotted mode}

While a mobile station employs antenna diversity, dual receiver method means that mobile station has two receiver branches, one receiving branch measures the signal strength and quality on the other frequency while another receiving branch is keeping on transmitting and receiving signals on the current frequency. This is especially suitable for antenna diversity in mobile station. However it is not easy to have antenna diversity in mobile station by normal antenna. Fortunately, the WCDMA system supports adaptive antenna technique development. It is possible that one of the beam formers of smart antenna in mobile station can be used to catch the signals to measure the signal strength and the quality on the other frequency while the other beam formers are used to maintain the connection on the current frequency.

The slotted mode method is used for inter- frequency handover measurement in WCDMA. By this way, the information transmitted are compressed in time domain to give a short Transmission Gap Length (TGL) of the channel to measure the signal strength on other frequency, The information are compressed by lowering the data rate from higher layers, or increasing the data rate by changing the spreading factor or reducing symbol rate by code puncturing or by changing the frequency rate. Fig.9 shows the basic mechanism of the slotted mode method.

It is more attractive that mobile stations don't need antenna diversity compared with dual receiver, but there is a trade off between system complexity and the advantage of this method. The inter-frequency handover is a Network Evaluated Handover (NEHO), and the decision algorithm is located in RNC.

\subsection{Inter-System between GSM and WCDMA}

WCDMA not only supports hard handover between different frequency carriers within WCDMA system,but also supports handover between WCDMA and other mobile communication systems which leads to the improvement of the coverage, capacity and balancing load of mobile communicatin. system, for example GSM,to offer a world coverage capacity. Mobile station should be multi-mode in order to support the inter-system handover. In addition the frame timing is critical for this type of handover. A WCDMA terminal can do the measurements either by requesting the measurement intervals in a form of slotted mode where there are break in the downlink transmission or then it can perform the measurements independently with a suitable measurement pattern. With independent measurements the dual receiver approach is used instead of the slotted mode since the GSM receiver branch can operate independently of the WCDMA receiver branch.

\section{CONCLUSIONS}

This paper presents a review the hard handover' soft/softer handover concepts in WCDMA system. Softer handover can improve the coverage capacity and system performance without increasing the system loading as it implements within the base stations. The soft handover is one of the important tools to improve the system performance, but it causes the system loading to increase, which is different than this softer handover. The parameters with possible soft handover algorithms are analyzed. The adding threshold, dropping threshold, timer-todropping are important parameters for soft handover algorithms. For different situation such as hot-spots, the parameters need to be adjusted dynamically to balance the network load or increase the coverage and system performance. Proper design of handover algorithms is important, since it has a great impact on the system performance and capacity. In packet transmission of WCDMA, soft handover gives the improvement of performance. Two important parameters, handover margin and maximum active size are analyzed.

In WCDMA, hard handover is necessary either for shared channel and common channel with intrafrequency or interfrequency to balance the network loads, or it is used to handover users to other systems such as GSM or IS-95 from WCDMA system.

\section{REFERENCES}

[1] D.K.Kim, and D.K. Sung,"Characterization of Soft Handoff in CDMA Systems," IEEE Proccedind, PP.1195-1202, 1999. 
[2] William C.y.Lee, "Mobile Cellular Telecommunications", $2^{\text {nd }}$ edition, McGraw Hill Inc-2000.

[3] H. Holma and A. Toskala,"WCDMA for UMTS, Radio Access for Third Generation Mobile Communication", Jhon Wiley \&Sons,Inc., 2000

[4] Yang,etal X; Ghaheri-Niri, S.;Tafazolli, R."Sectorization gain in CDMA cellur systems", 3G Mobile Communication Technologies, pp. 70-75, 2000

[5] X.Yang, and S.G.Niri and R. Tafazolli,"Evaluation of Soft Handover Algorithms for UMTS", IEEE Procceding, pp 772-776,2000.

[6] Hiltunen, K., Binucci, and N.; Bergstrom, $\mathrm{J}$,"Comparison between the periodic and eventtriggered intra-frequency handover measurement reporting in WCDMA", IEEE Wireless Communications and Networking Conference, pp.471-475, 2000.

[7] N.Binucci, K. Hiltunen and M. Caselli,"Soft handover gain in WCDMA ", Vehicular
Technology Conference .2000.IEEE VTS 2000.Vol.3.pp1467-1427.

[8] J.luo.m .Dillinger and E.Schulz,"Research on timer setting for soft handover algorithm with different system loads inWCDMA", Communication Technology Proceedings, Vol.1. Pp741-747.2000.

[9] K. Sipila, M. JASBERG, J.Laiho-Steffens and A.Wacker,"Soft handover gains in fast power controlled WCDMA uplink", Vehicular Technology Conference, 1999, IEEE, Vol.2 .pp 1539-1543, 1999.

[10] R. Pasarad and T.Ojanpera,"An Overview of CDMA Evolution toward Wideband CDMA", IEEE Comm.Servey, Vol.1, No.1, 1998.

[11] S.De Capitani Di Vimercati,"Mobile agent Technology for Remote Measurement,IEEE Transaction on Instrumentation and Measurement, Vol.55, No.5, pp 1559-1565, October 2006.

[12] Internet websites.

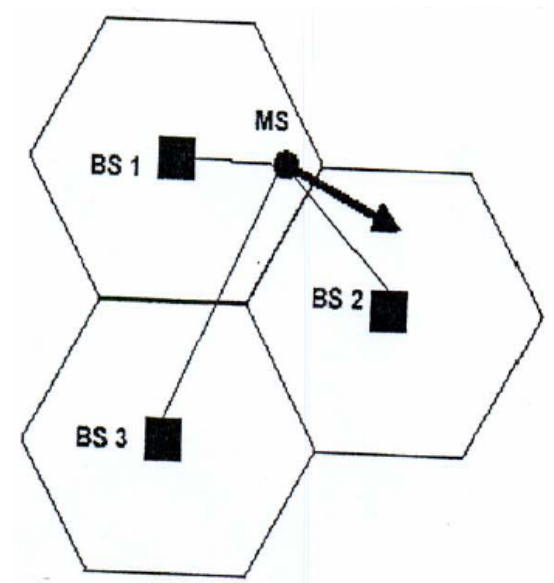

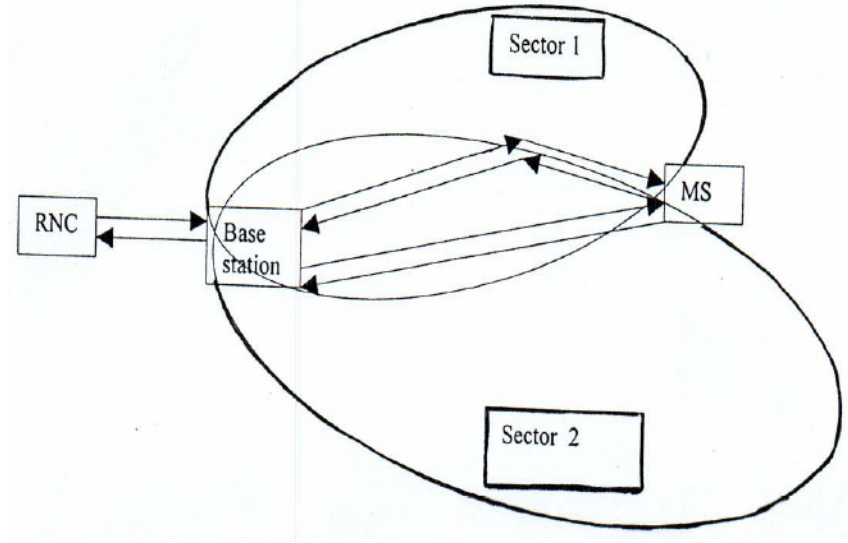

Figure (2) Softer Handover

Figure (1) Soft Handover in WCDMA System

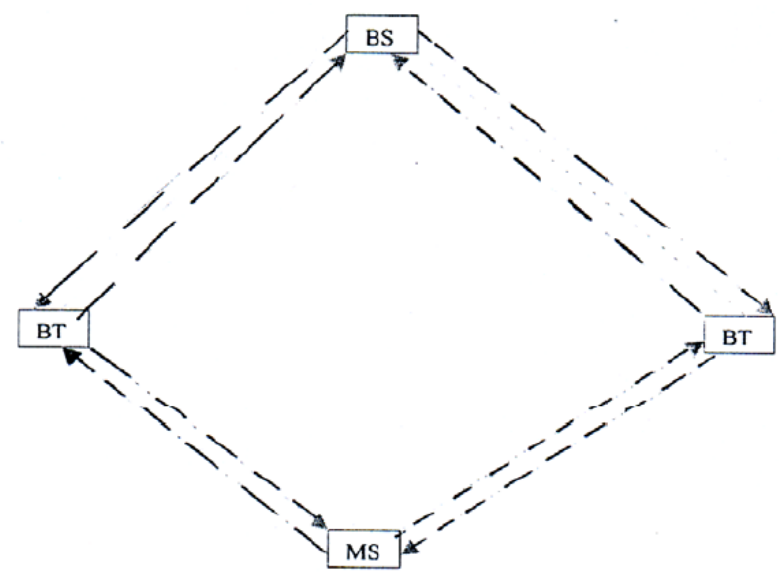

Figure (3) Soft Handover with Two Base Stations 
Raad H. Thaher, "Noise Reduction in CDMA System Using Soft Handover"

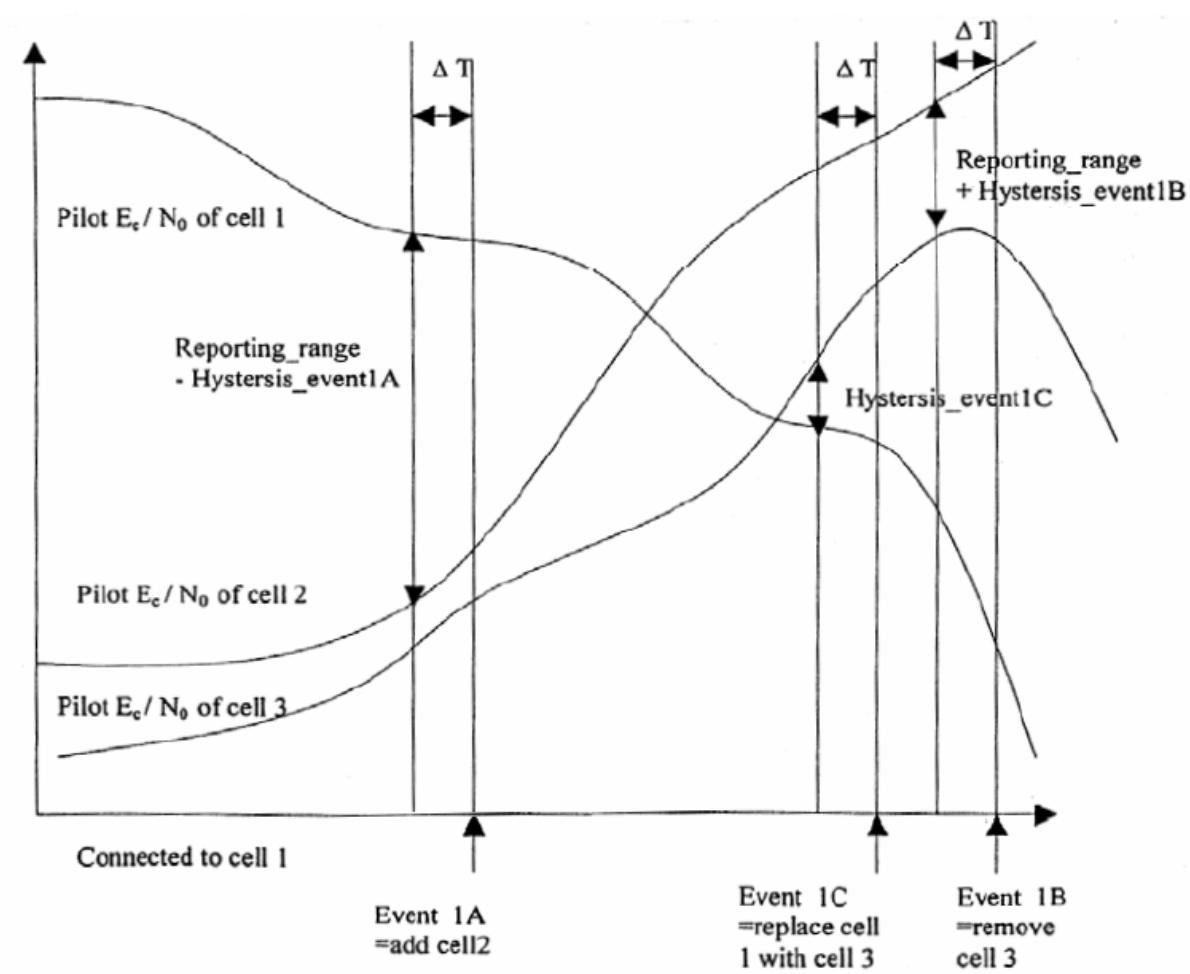

Figure (4) General Scheme of WCDMA Soft Handover Algorithm

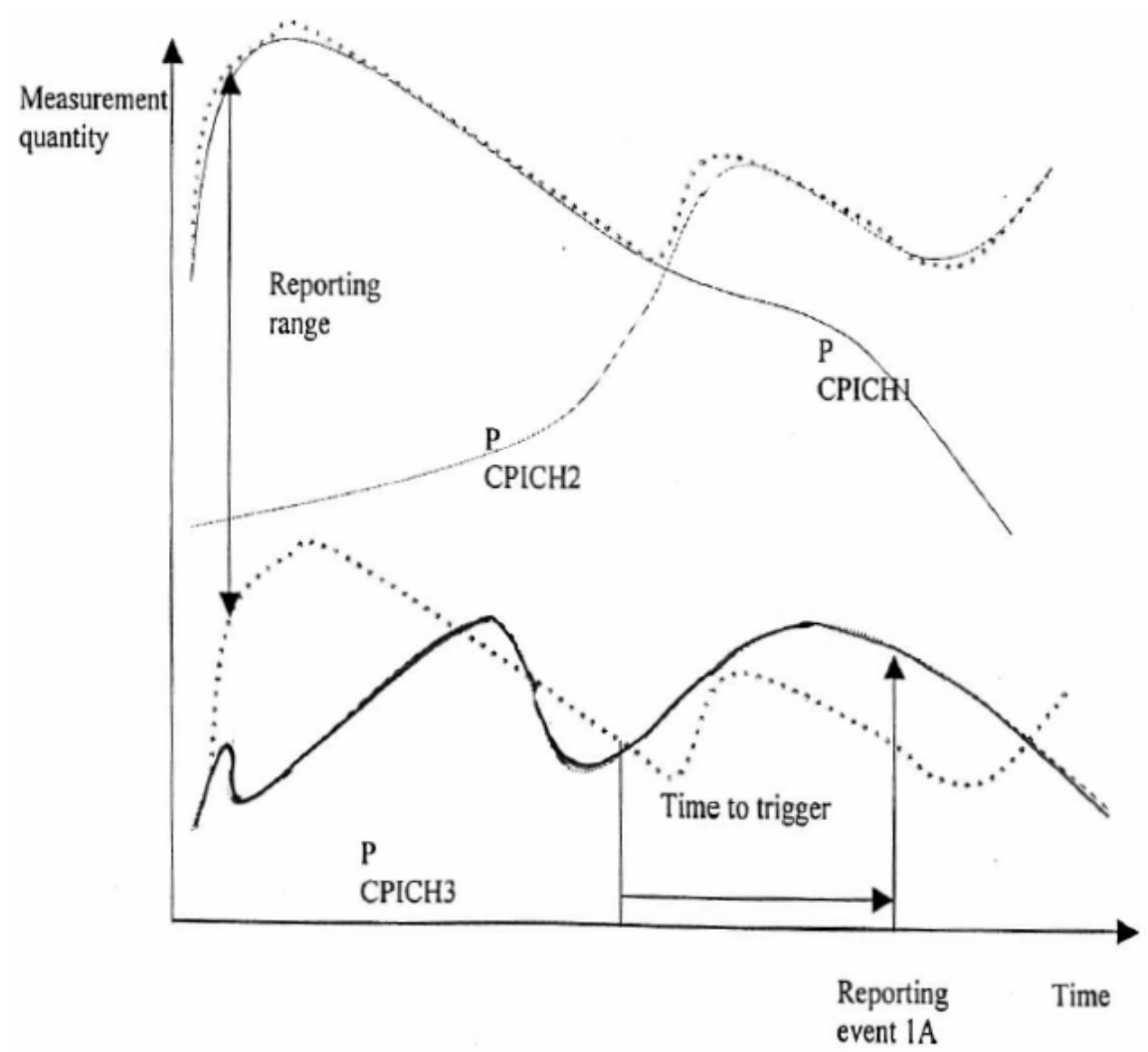

Figure (5) Time-to-Trigger Limits the Amount of Measurement Reports. 


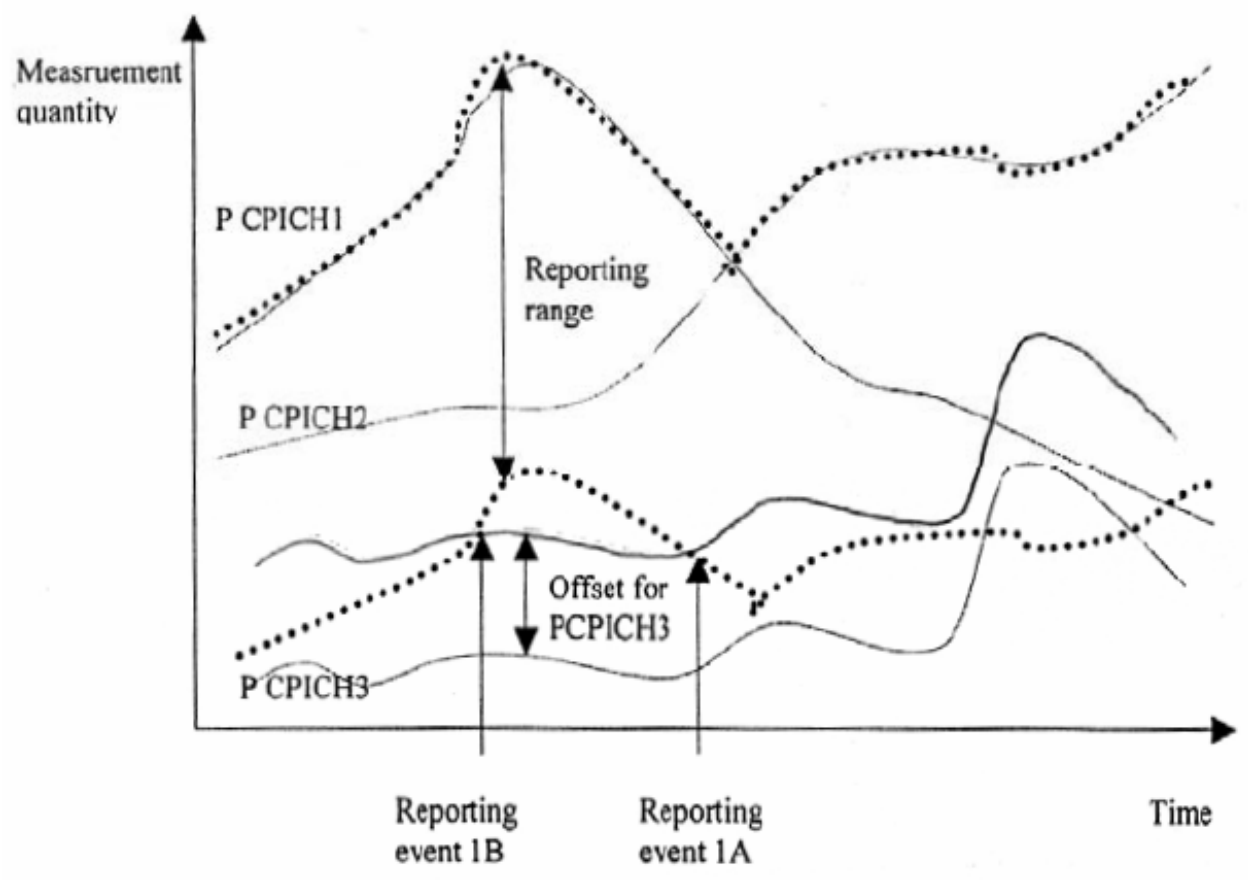

Figure (6) Positive Offset Applied to Primary CPICH3 Before Event Evaluation in the User Equipment.

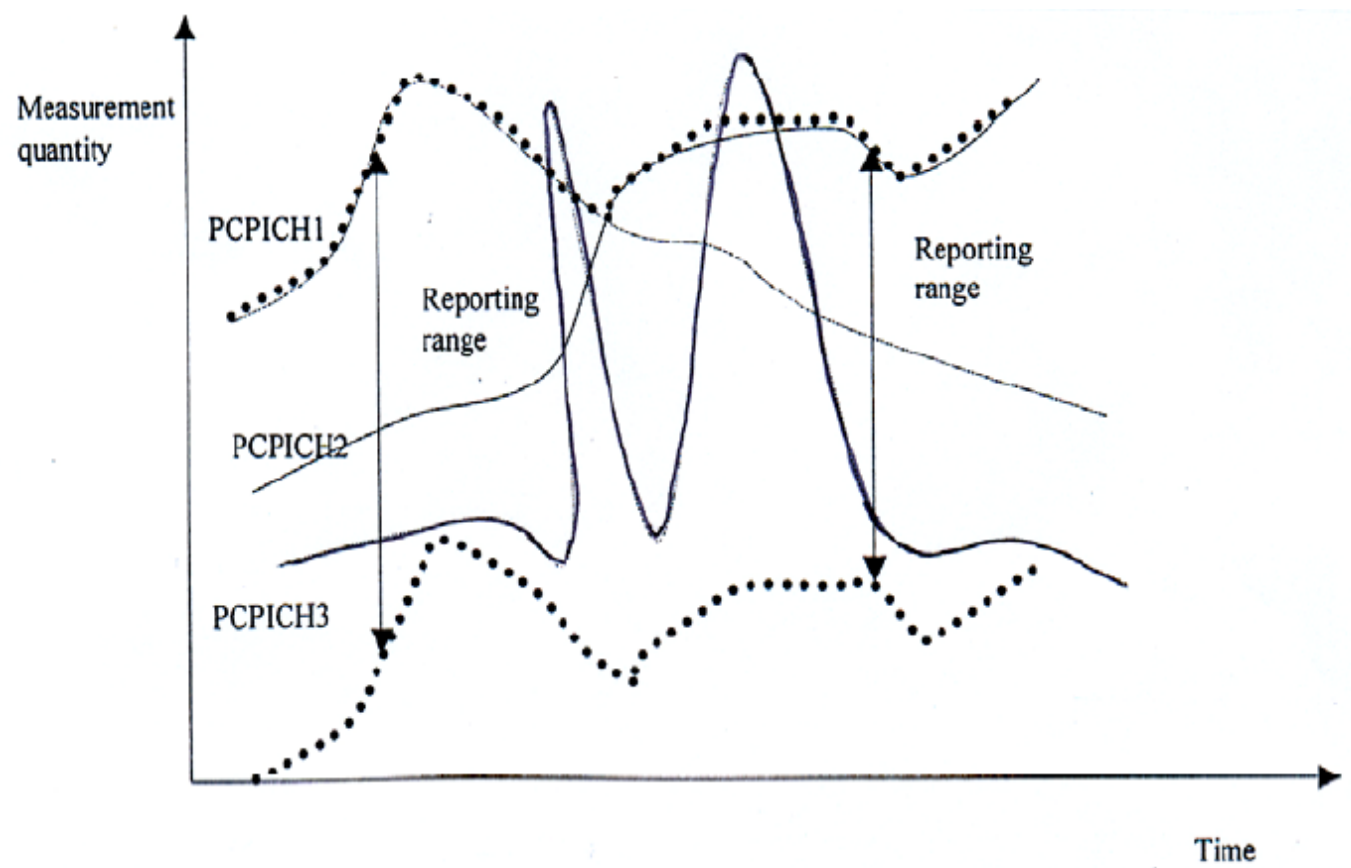

Figure (7) Mechanism for Forbidding a Neighboring Cell to Affect the Reporting Range to Extend its Border 


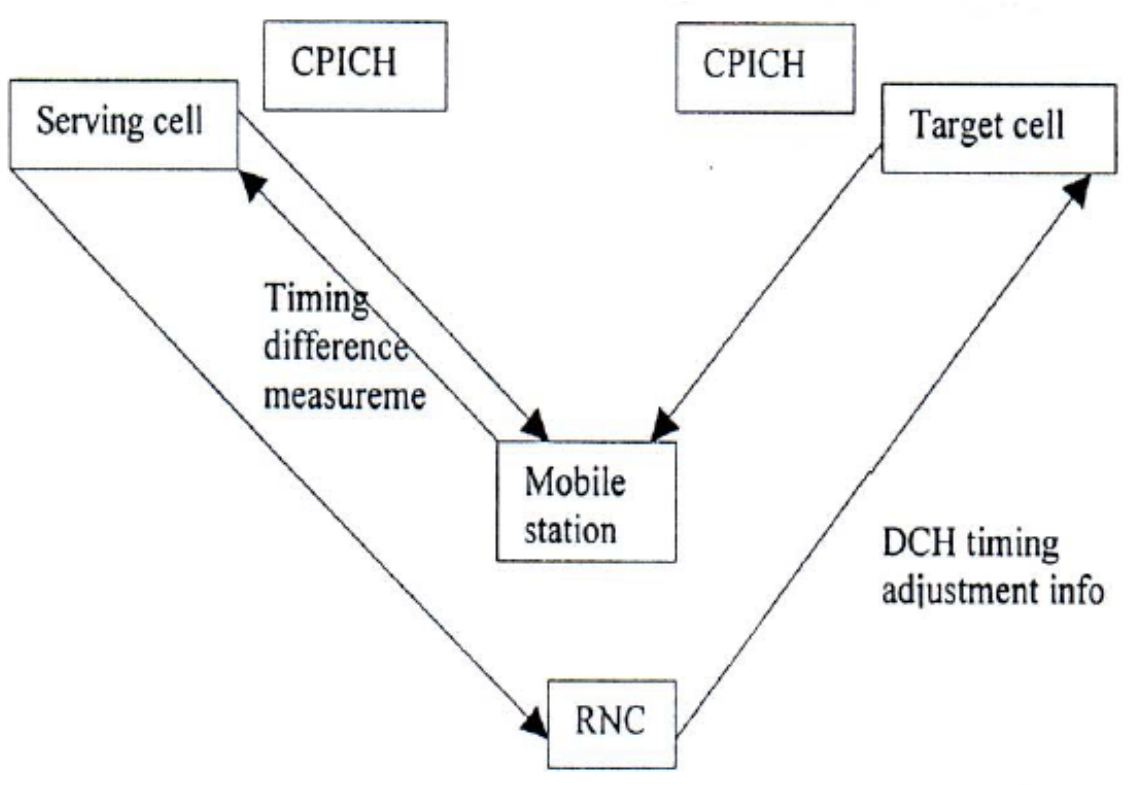

Figure (8) Timing Measurement for Soft Handover.

power

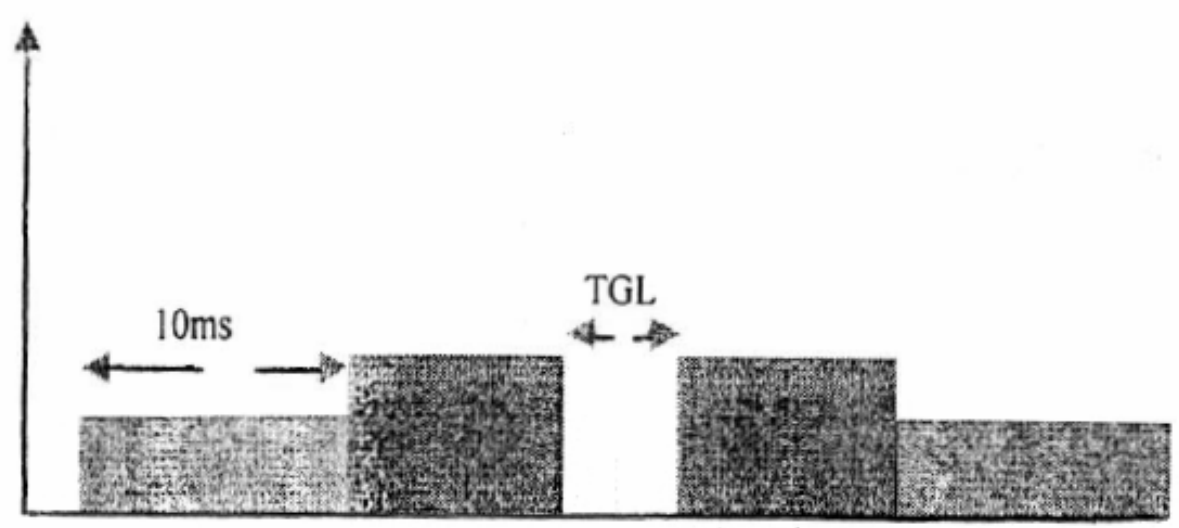

Figure (9) Frame Boundary. 